\title{
Instituciones y desarrollo municipal: un análisis multinivel
}

\section{Institutions and municipal development: a multilevel analysis}

\author{
Henio Millán-Valenzuela* \\ Martha Paola García-PÉrez**
}

\begin{abstract}
The importance of institutions has been an answer to the search of deep causes of development, beyond economic factors traditionally invoked. To prove the superiority of those variables over the later ones, seven multilevel models applied to Mexican municipalities are developed. It is concluded that democratization, criminal justice and government system, and innovation institutions surpass labor productivity, investment, and technical progress, when explaining the differences on municipal levels of development.
\end{abstract}

Keywords: institutions, development, municipal, multilevel.

\section{Resumen}

La importancia de las instituciones ha sido una de las respuestas a la búsqueda de las causas profundas del desarrollo, más allá de los factores económicos tradicionalmente invocados. Para probar la superioridad de aquellas variables sobre estos últimos, se desarrollan siete modelos multinivel aplicados a los municipios mexicanos. Se concluye que la democratización, el sistema de justicia y gobierno, así como las instituciones de innovación superan a la productividad, a la inversión y al progreso técnico al explicar las diferencias en los niveles de desarrollo municipal.

Palabras Clave: instituciones, desarrollo, municipal, multinivel.

* Investigador de El Colegio Mexiquense, A. C. México, correo-e: hmillan@cmq.edu.mx

** Estudiante de la maestría en ciencias sociales con especialidad en desarrollo municipal de El Colegio Mexiquense A. C. correo-e: garciaperezpaola@gmail.com 


\section{Introducción}

Desde la llamada década perdida, en México, propiciar un clima de estabilidad económica se volvió el objetivo primordial de las políticas públicas, no obstante, se omitió la promoción del desarrollo o se delegó esta responsabilidad a los gobiernos locales. Una extensa literatura relacionada con estudios económicos, sociológicos y territoriales concluyó que en el territorio latinoamericano era posible aplicar una serie de fórmulas, tales como: "pensar globalmente y actuar localmente"; "descubrir vocaciones económicas regionales"; "crear ventajas competitivas y vernáculas"; "desarrollar distritos industriales y polos de crecimiento"; complementar actividades y fomentar "clusters"; y erigir a los gobiernos locales como coordinadores de una "gobernanza" que promovía asociaciones entre universidades y empresas, así como entre éstas y sus proveedores para integrar más eficientemente las cadenas productivas. Todas ellas fueron algunas de las fórmulas recomendadas, y adoptadas, por muchos gobiernos que operan al nivel sub-nacional.

De esta forma, se cerraba un círculo que especifica las bases de las políticas neoliberales con respecto al desarrollo, consistente en la renuncia a un Estado nacional, promotor del desarrollo, y la transferencia de esta responsabilidad a los gobiernos locales. El primero debería favorecer la conservación del equilibrio macroeconómico y promover políticas sociales subsidiarias, mientras los segundos tendrían la encomienda de atraer inversiones, promover innovaciones y elevar la productividad, mediante acciones amigables y en línea con la lógica del mercado. Esto implicó cambios en el marco institucional, que no siempre fueron homogéneos ni en su diseño ni en su implementación.

El objetivo del presente texto es analizar la influencia del marco institucional en el desarrollo local, es decir, en el municipio. En la primera sección se exponen brevemente las ideas neoinstitucionalistas que integran el aparato teórico sintetizado del documento. De manera general, en el texto se despliega un modelo de análisis multinivel, en el que se asume el Índice de Desarrollo Humano (IDH) como proxy del desarrollo municipal, ubicado en primer nivel, mientras las variables institucionales nutren el segundo, que se ha ubicado en orden estatal. Finalmente, se plantean las conclusiones de este estudio.

\section{Instituciones como determinantes del desarrollo}

La recuperación del tema del desarrollo, tras la obsesión por la estabilidad macroeconómica, no vino sola sino trajo consigo la inquisición sobre sus causas más profundas. No bastaba ya con subrayar la importancia de la 
acumulación de capital (Ricardo, 1817 y1998), la tasa de ahorro y la productividad asociada al progreso técnico (Solow, 1956). Ahora las preguntas eran ¿por qué unas sociedades invierten más que otras? ¿son más productivas e innovan más aceleradamente?

Las respuestas, aunque variadas, se pueden aglutinar en dos campos exegéticos: las explicaciones geográficas y las neoinstitucionalistas (Rodrik et al., 2002). Las primeras, a cargo de Jeoffry Sacks y sus colegas, enfatizan las condiciones climáticas y la localización como determinantes directos del crecimiento económico, así como su influencia en la selección de instituciones y de políticas, especialmente, las comerciales. Con relación a los factores directos, encuentran que la geografía transmite sus efectos a través de los costos de transporte, la productividad agrícola y el peso de las enfermedades; a lo que agregan la influencia sobre la densidad demográfica y el crecimiento de la población (Gallup et al., 1998).

En el otro polo, las propuestas neoinstituionalistas experimentaron una creciente atención de los analistas, hasta casi opacar la visión geográfica. La clave residió en la introducción de dos ingredientes que parecen poblar la abigarrada constelación de sus feligreses: la importancia de la historia y de la política en el curso del desarrollo de las naciones.

La introducción de la historia es quizá una forma de recuperar uno de los principales aportes de las teorías del crecimiento endógeno: la determinación del curso del desarrollo por las condiciones iniciales y, en consecuencia, el abandono de la idea de convergencia absoluta (Romer, 1994; Lucas, 1988) que sostuvieron los modelos neoclásicos tradicionales que vaticinaban que con tasas de ahorro y de crecimiento de la población similares, las economías más rezagadas convergerían a un mismo nivel de bienestar que las más avanzadas (Solow, 1956).

El frente neoinstitucionalista acogió este abandono conceptual mediante el examen de las instituciones que habían propiciado distintas variantes de la conquista y de la colonización (Acemoglu et al., 2001) y mediante la introducción de la dependencia de la trayectoria o path dependence (North, 1993). Ahí donde las condiciones climáticas eran benignas; la población, dispersa; y las tasas de morbilidad, bajas, se desarrollaron instituciones de calidad, en respuesta al deseo de expandir las poblaciones de origen europeo. Estas instituciones apuntalaron una mayor igualdad e inclusión social, sistemas más democráticos y una protección más vigorosa de los derechos de propiedad, así como un mayor respeto por el orden legal. En cambio, ese deseo de establecimiento europeo permanente no se experimentó en zonas colonizadas en las que la población, además de concentrada, sufría altas tasas de mortalidad y morbilidad. En estas áreas, las instituciones instauradas fueron de carácter extractivas, encaminadas a explotar a los nativos. Por tal razón, giraron 
en torno a la conservación de la desigualdad y exclusión social, un sistema legal ineficiente y derechos de propiedad, no sólo escasamente protegido, sino orientados a la extracción de rentas.

La diferencia entre instituciones rentistas e instituciones favorables al desarrollo productivo -extractivas vs. inclusivas- (Acemoglu y Robinson, 2012) ha sido una de las claves del neoinstitucionalismo para articular un mecanismo de transmisión entre instituciones y desarrollo. Desde los trabajos tempranos de North (1984, 1993), los derechos de propiedad ocuparon un lugar central en la explicación de los cursos divergentes de las naciones: aquellas que los especificaban claramente y los defendían vigorosamente y, sobre todo, los estructuraban en un sentido moderno, inclinado a vincular el bienestar sola o privilegiadamente al esfuerzo individual, que debería desplegarse en un marco razonablemente competitivo. Este fue el caso de Inglaterra a partir del siglo xvi. En cambio, España estructuró derechos de propiedad, no sólo difusos e intermitentemente expoliadores, sino también de índole monopólico, con la finalidad de asegurar rentas para afrontar las crecientes necesidades de recursos de una Corona dispendiosa y en crisis fiscales casi permanentes.

La influencia nociva de las rentas sobre la acumulación de capital, la productividad y la inversión ya la había establecido la economía política clásica, especialmente mediante el trabajo de Ricardo (1817 y 1998), en su combate de las ideas mercantilistas y fisiocráticas (Millán, 2012). En primer término, en la medida en que la renta de la tierra evolucionaba en sentido contrario de la productividad laboral, los terratenientes podían mantener una postura adversa a la eficiencia y el progreso técnico. En segundo lugar, la tendencia creciente de la renta de la tierra se expresaba en salarios ("naturales" y de mercado) en ascenso, que acababan impactando negativamente a la tasa de ganancia y, por esta vía, la inversión y crecimiento de una nación.

A esta visión sobre la tasa de crecimiento de largo plazo se sumó otra aportación con tintes novedosos. Cuando las instituciones no funcionan adecuadamente y son ineficientes para garantizar los derechos de propiedad, reforzar los contratos, suministrar información suficiente y, en general, para reducir los comportamientos oportunistas, la economía se plaga de significativos costos de transacción que desvían beneficios y costos sociales de los privados. Es decir, que producen sistemas ineficientes en el sentido de Pareto. De esta forma, se cierra el círculo teórico del mecanismo de transmisión entre instituciones y economía eficiente (el corto plazo), por un lado, y en crecimiento (el largo plazo), por el otro.

Lo que Ricardo (1817 y 1998) vio, y los neoinstitucionalistas generalizaron, fue una estructura de poder articulada para mantener los privilegios, fuente de toda actividad rentista, que permitía "extraer" una parte 
del producto social sin trabajar o invertir; es decir, sin aportar nada para su generación, con la única legitimidad que la que suministra un derecho que no es extensible a todos los demás. Esa estructura corresponde, precisamente, a las instituciones "extractivas". A ellas, Acemoglu y Robinson (2012) contraponen las instituciones "inclusivas", que corren históricamente por otra pista. Mientras que las primeras se activaron y arraigaron en áreas colonizadas en las que fue posible encontrar grupos sociales nativos susceptibles de ser explotados y, por tanto, propicias para desligar el bienestar de las clases dominantes del esfuerzo, la innovación y la inversión; las segundas, evolucionaron a partir de que la supervivencia de los colonizadores dependió crucialmente de los esfuerzos desplegados por ellos.

Cada una de estas clases de instituciones reflejaban y estaban inscritas en dos tipos de estructura de poder. Donde prevalecieron las instituciones extractivas, se desarrollaron otras instituciones de naturaleza excluyente, tanto en ámbito social como en el político, porque ese marco institucional (ya sea de manera formal o informal) era el necesario para mantener viva la desigualdad sobre la que se basaba la explotación de la mayoría de la población. De esa estructura derivaron gobiernos de corte autoritario que marcaron el posterior devenir de las zonas subdesarrolladas y, al mismo tiempo, dificultaron la reversión de las condiciones iniciales.

En el otro extremo, la penuria seminal de los primeros colonizadores, así como su imposibilidad de explotar a los grupos nativos, derivaron en instituciones inclusivas, no sólo en el plano económico, sino también proclives hacia una mayor igualdad social e integración política del pueblo llano, que desembocaron en regímenes de índole democrática, con todos sus atributos: libertades civiles, derechos políticos, apego a la ley y protección eficiente de unos derechos de propiedad que estimulaban la competencia.

La simbiosis entre instituciones económicas y políticas fue otro de los aportes del nuevo institucionalismo. El examen más escrupuloso-aunque a veces, no suficientemente claro- de North (1993) se distinguió por otorgar a la política el lugar de principal determinante del desarrollo. En primer término, nos ofrece una definición clara de las instituciones como reglas que estructuran el comportamiento humano y cuya función fundamental es reducir la incertidumbre en todos los tipos de intercambios humanos. Su influencia sobre la conducta se ejerce a través de la estructuración de incentivos positivos y negativos, que orientan las posibilidades y las restricciones de la acción individual y colectiva. En el ámbito económico y político, las instituciones determinan qué, cómo y cuándo se puede ganar; y por esta vía, delinean las ventanas de oportunidades que las organizaciones -entidades distintas de las instituciones- aprovechan 
para erigirse y desarrollarse, así como para pugnar por cambios institucionales que se experimentan de forma incremental.

De esta forma, el marco institucional inicial determina el tipo y la conducta de las organizaciones; pero éstas se convierten en el principal agente de cambio de ese encuadramiento, cuando perciben que una o varias modificaciones pueden alterar a su favor las oportunidades hasta entonces establecidas. De esta mecánica deriva la dinámica del cambio institucional y, por ende, del desempeño económico y social. La trayectoria puede derivar en una sociedad desarrollada y democrática o en otra de estancamiento y subdesarrollo de corte más o menos autoritario. Todo depende del marco institucional: si las reglas del juego son favorables a la competencia y la apropiación de los frutos del esfuerzo de quienes lo despliegan, el resultado será la primera vía; si, en cambio, están estructuradas para defender privilegios y actividades rentísticas (señaladamente, las monopólicas), la segunda ruta.

En esto hay una coincidencia plena de Acemoglu y sus colegas con el enfoque seminal de North. También la hay en la proposición de que el marco institucional es invariablemente impuesto por las élites, en un afán de articular una estructura de poder que proteja y promueva sus intereses. La clave de la divergencia en el desempeño económico reside en que, en un caso, esos intereses son expresados mediante instituciones que promueven el desarrollo, mientras que en el otro, no.

Sin embargo, el análisis del Premio Nobel es más sutil, al exponer una visión más elaborada de la influencia de la política en el desarrollo, así como un rol significativo para las organizaciones, la ideología y la dependencia de la trayectoria. Por razones de espacio no es posible exponer la forma en que se entreveran estas tres concepciones. Bástenos, por ahora, señalar tres conclusiones del análisis: 1 . No existen razones para que opere un darwinismo institucional, en el que las instituciones "buenas" sustituyen, mediante ensayo y error, a las "malas", como parece insinuar Alchian (1950) y, en general, cualquier enfoque basado en expectativas adaptativas; 2. La persistencia de las instituciones "malas" obedece a la resistencia de las élites, pero también del papel de las organizaciones como creaciones y agentes de cambio del marco institucional. En éstas se configuran un tipo de habilidades y de visiones del mundo (ideología), que disponen la forma en que se percibe la ventana de oportunidades y las capacidades para aprovecharlas; y 3 . En este sentido, el pasado determina el presente, y éste el futuro. El pasado especifica las opciones, la forma de percibirlas y la capacidad para emprenderlas exitosamente.

En eso consiste la dependencia de la trayectoria, que decide el curso futuro del desarrollo mediante las visiones y capacidades heredadas del pasado. En términos generales, y lejos de un determinismo absoluto de 
la historia, las nuevas instituciones tienden a reproducir muchos de los elementos esenciales de sus antecesoras, con la consecuente reproducción de los incentivos a favor o en contra del desarrollo, según sea el punto de partida.

Aunque Acemoglu y Robinson (2012) y North (1984 y 1993) coincidieron en la importancia de los gobiernos para asegurar el estado de derecho y de la democracia, entre ellos es perceptible una diferencia de énfasis. Para el segundo, el Estado tiene como función principal proteger y estructurar los derechos de propiedad, señaladamente el reforzamiento de los contratos; mientras que para los primeros, el énfasis recae en la constitución de regímenes democráticos. Éstos no sólo aseguran el imperio de la ley, sino, además, distribuyen el poder a favor de la mayoría ciudadana, lo que permite controlar al Estado y delimitar la esfera de influencia y la actuación interesada de las élites. Esta distribución del poder es el mecanismo para la inclusión y la equidad sociales.

Tal es el énfasis en la democracia y sus atributos incluyentes, que les anima a pronosticar el desarrollo a partir de los regímenes políticos: a) aquellas sociedades que no logran consolidar un Estado -y por ello una fuerza legítima que imponga la ley-, nunca se desarrollarán; b) las que logran conformarlo, pero bajo esquemas autoritarios, experimentarán periodos de crecimiento, pero éste tenderá a agotarse después de un periodo determinado, hasta incurrir en el estancamiento; y c) sólo las sociedades democráticas, en las que son indispensables las instituciones inclusivas, lograrán el desarrollo mediante tasas de crecimiento de largo plazo sostenidas y mejoras continuas en un bienestar social creciente y equitativo.

En resumen, las dos vertientes del neoinsitucionalismo enfatizan el papel del Estado en el desarrollo, ya sea en su carácter de estructurador y garante de las reglas legales, o en el grado en que refleja el poder ciudadano. Por tal razón, los indicadores diseńados para el ejercicio cuantitativo que será abordado en las siguientes secciones, incluyen una variable institucional que da cuenta de los gobiernos y el sistema de justicia, y otra del grado de democratización de las entidades federativas. Además, se agrega un indicador institucional para cubrir, bajo el manto de la innovación, las capacidades y visiones de las organizaciones empresariales.

\section{Productividad, inversión y progreso técnico como determinantes}

Los modelos multinivel han experimentado una creciente acogida entre los científicos sociales inclinados a la medición. La razón estriba, en buena parte, en que constituyen un expediente eficaz para capturar la diversidad y, de esta manera, para escapar de los engañosos promedios homogeneizadores. Y los municipios mexicanos son diversos y atravesados por 
una gran variedad de componentes idiosincráticos. Por tal motivo, esta sección y la siguiente se abocan a especificar y estimar un grupo de modelos jerárquicos, con la finalidad de explicar la disparidad de los niveles de desarrollo municipal en función de sus determinantes más inmediatos e invocados -la inversión, la productividad laboral y el progreso técnicopara posteriormente examinar la influencia que ejercen las instituciones en esta vinculación. De esta forma, se cubren las dos "capas" analíticas por las que transcurren las preocupaciones en torno al desarrollo: las causas directas y las profundas, tal y como se expuso en la introducción.

Para tales efectos, se seleccionó aleatoriamente una muestra representativa de 482 municipios, con un error muestral del 4\%. Nuestro modelo opera con dos niveles: el municipal y el estatal. En el primero, se selecciona al IDH municipal como variable dependiente y proxy del desarrollo municipal; y a la productividad media laboral, la inversión acumulada (dotación de activos por trabajador) y al progreso técnico, aproximado por la productividad total de los factores, como variables independientes o determinantes inmediatos del desarrollo municipal, en concordancia con la teoría convencional. La información proviene de PNUD, 2010, y del Censo Económico de 2009, publicado por Inegi.

En el segundo nivel se introducen las variables institucionales, compuestas por tres Índices: Democratización, Justicia y Gobierno e Innovación, construidos con los datos que el Instituto Mexicano de la Competitividad (IMCO, 2014) utilizó para estimar los indicadores de competitividad de las entidades federativas de 2010. Todas las variables independientes - primer y segundo nivel- han sido "centradas", con el propósito de que el intercepto o constante de la regresión refleje la media general de todos los municipios (Pardo et al., 2007). ${ }^{1}$

\subsection{El modelo vacío: las discrepancias entre los IDH municipales}

Cuando se examina de un vistazo los IDH municipales, es fácil percibir que entre ellos existen diferencias notables. ¿Son estas diferencias estadísticamente significativas u obedecen a un problema de percepción? Los modelos vacíos, que constituyen el primer acercamiento de los modelos multinivel al estudio de la diversidad, tratan de contestar este tipo de interrogantes. Su característica reside en que son expresiones que deliberadamente excluyen las variables independientes o explicativas. Son, en este sentido, una variante del análisis de varianza (ANOVA), en la medida en que después de constar (o rechazar) que las diferencias son significativas,

\footnotetext{
${ }^{1}$ El procedimiento para "centrar" la media consiste en restar a cada variable independiente su media respectiva
} 
se aventura a una primera estimación entre variaciones explicadas por diferencias inter (entre estados) e intragrupales, es decir, entre los municipios dentro de los estados.

En nuestro caso, el modelo vacío se especifica de la siguiente manera:

$$
\begin{array}{ll}
I D H_{i j}=\beta_{o j}+e_{i j}(2.1) & \text { Nivel 1 } \\
\beta_{o j}=\gamma_{00}+u_{o j}(2.2) & \text { Nivel 2 }
\end{array}
$$

La intención de esta primera especificación es hacer de los IDH una variable que contiene solamente efectos fijos y aleatorios. Los primeros corren a cargo de $\gamma_{00}$, la media muestral o gran media, que representa un componente común de todas las medias estatales de los IDH $\left(\beta_{o j}\right)$. Los efectos aleatorios complementan la información, en la medida en que están dirigidos a detectar las diferencias entre los estados, por un lado, y las que prevalecen entre los municipios de una misma entidad federativa. De acuerdo con el ANOVA, las primeras se expresan como distancias entre las medias estatales y la muestral $\beta_{o j}-\gamma_{00}$ mientras las segundas como desviación de un IDH particular con relación al promedio estatal $\left(I D H_{i j}-\beta_{o j}=e_{i j}\right)$.

El modelo mixto nulo combina los dos niveles y se expresa, por tanto, de la siguiente manera:

$$
I D H_{i j}=\gamma_{00}+\left(u_{o j}+e_{i j}\right.
$$

De esta forma, el IDH municipal queda exclusivamente en función de sus efectos fijos y aleatorios, es decir, de sus componentes comunes y diferenciados. Las estimaciones de ambos se consignan en los cuadros 1 y 2, bajo el rubro de "modelo vacío" (al final del texto).

Como se aprecia, la media muestral o gran media de los IDH municipales es de 0.8160 , según lo consigna la intersección estimada en el cuadro de efectos fijos. Esta estimación es estadísticamente significativa, como lo avalan la prueba t y el nivel de significancia correspondiente. Otro tanto sucede con la varianza de los residuos y la del término aleatorio, si juzgamos por el valor del coeficiente Wald Z y la significación asociada. De esta forma, los cálculos confirman la existencia de elementos comunes (la media general) y diferenciados, que nos advierten que las discrepancias entre los IDH pueden asumirse como estadísticamente válidas.

Estas discrepancias, entonces, obedecen tanto a diferencias entre los estados como a las que se registran dentro de los mismos. ¿Qué tanto se le puede atribuir a unas y a otras? La respuesta nos la brinda el Coeficiente de Correlación Interclase (CCI): 


$$
C C I=\frac{\sigma^{2} u_{0 j}}{\sigma^{2} u_{0 j}+\sigma^{2} u_{e i j}}=\frac{0.00142}{(0.001420+0.003355)}=0.3662
$$

El resultado nos informa que 36,62\% de las diferencias en los IDH obedecen al factor "estado" (es decir, a las de carácter interestatal); mientras que $63,38 \%$, a las que operan en los municipios dentro de un mismo estado. Por tanto, es posible concluir que la heterogeneidad en el desarrollo municipal no sólo responde a las diferencias regionales invocadas frecuentemente (norte-centro $v$ s. sur-sureste), sino también -y fundamentalmente-a disparidades profundas al interior de los estados que aglutinan esas regiones. En otros términos, el desarrollo no sólo tiende a concentrarse en ciertas entidades de la república, sino también en algunos municipios que éstas albergan. En este sentido, el desarrollo no sólo es desigual en términos interestatales, sino -sobre todo- de forma intraestatal. Esta última desigualdad se revela como la más importante, a la hora de explicar la disparidad total.

\subsection{La productividad laboral y las diferencias entre los IDH municipal: modelo 2}

Si el ejercicio anterior avaló la existencia de discrepancias estadísticamente significativas entre los IDH, y que éstas ocurren tanto entre los estados como al interior de los mismos, surge la pregunta sobre cuáles son las causas de tales diferencias. El modelo que se describe en esta sección argumenta una primera respuesta, que aspira a ser complementada en la próxima.

La productividad laboral es la principal determinante de las remuneraciones laborales. Sin embargo, la variable escogida es la productividad media (PML), y no la marginal, en virtud de varias razones teóricas y metodológicas. ${ }^{2}$ La proxy es la relación entre la producción bruta (PB) y la cantidad de personal ocupado (L), centrada por su media: ${ }^{3}$

\footnotetext{
${ }^{2}$ En primer lugar, la igualación de los salarios al valor de la productividad marginal supone precios y salarios dados para las empresas individuales, lo que a su vez implica competencia perfecta. Este supuesto está lejos de cumplirse en las estructuras de mercado mexicano. En segundo término, como ha demostrado la teoría del oligopolio moderno (Koutsoyianis, 1985), las empresas suelen mantener cierta capacidad de reserva que hace que la productividad media se vuelva constante, una vez que se alcanza la escala mínima óptima. A partir de este nivel, es igual a la productividad marginal. En tercer lugar, los datos censales no facilita el cálculo de la productividad marginal.

${ }^{3}$ En términos estrictos, la productividad media laboral debería de ser la relación entre el valor agregado y el personal ocupado. Sin embargo, se optó por la producción bruta, en virtud de que frecuentemente los municipios registran valores agregados censales negativos, que revelan que la cuantía de los insumos es mayor que la producción bruta. Esto puede deberse a un problema contable, que registra los inventarios de bienes intermedios como si hubieran sido utilizados en la pro-
} 


$$
P M L=\frac{P B}{L}
$$

De esta forma, el modelo 2 se especifica de la siguiente manera:

$$
\begin{aligned}
& I D H_{i j}=\beta_{o j}+\beta_{1 j} \mathrm{PML}_{i j}+e_{i j} \text { (2.1) Nivel } 1 \\
& \beta_{o j}=\gamma_{00}+u_{o j} ; \beta_{1 j}=\gamma_{10} \quad \text { (2.2) Nivel } 2
\end{aligned}
$$

En este caso, tanto $\beta_{o j}$ como $\gamma_{00}$ siguen representando a las medias estatal y muestral de los IDH, respectivamente; pero ahora se trata de parámetros condicionados a que el PML adopte su valor promedio. A ello se agrega que el coeficiente de respuesta de los IDH a la PML (la pendiente) se asume fijo $\left(\beta_{1 j}=\gamma_{10}\right)$ y, por tanto, común a todos los municipios. Esto equivale a igualar en todos ellos la PML; y en todos los estados, la relación que la vincula con el IDH. Es decir, anula las diferencias en esta última variable que obedecen a este factor, así como la influencia que ejerce. Se trata, en el fondo, de un contrafactual que permite estimar, posteriormente, esa influencia. ${ }^{4} \mathrm{El}$ modelo mixto, por su parte, se expresa de la siguiente manera:

$$
I D H_{i j}=\gamma_{00}+\gamma_{01} \mathrm{PML}_{i j}+\left(u_{o j}+e_{i j}\right)
$$

La estimación de los efectos fijos de este modelo se encuentra en el cuadro 1, y la de los efectos aleatorios, en el cuadro 2; en ambos casos bajo el rubro de "modelo 2". Todos los parámetros de ambos tipos de efectos resultaron significativos. ${ }^{5}$ En la sección de efectos fijos, resaltan la ligera disminución del IDH promedio de toda la muestra (la gran media), en virtud de que ahora está condicionada, y que a una mayor PML corresponde un IDH más alto. Específicamente, por cada millón pesos de diferencia en la productividad municipal, el IDH diferirá en 0,027 puntos; es decir, en 3,31\% del promedio muestral incondicionado.

Los cambios más importantes de la introducción de la PML se aprecian en los efectos aleatorios. Por un lado, la varianza de los residuos se ha reducido en $1,9 \%$. Esto significa que las diferencias en la productividad

ducción. Por otro lado, los valores censales negativos implican productividades laborales (de capital) negativas. Ello significaría que el empleo disminuye el producto final, en lugar de aumentarlo.

${ }^{4} \mathrm{El}$ contracfactual trata de responder a la siguiente pregunta: ‘a cuánto equivaldrían las diferencias entre los IDH municipales, si su productividad fuera igual en todos ellos y la respuesta de aquellos a esta última fuera la misma en todos los estados? Si la respuesta es contrastada con el valor actual de las discrepancias, se determina la influencia de la PML.

${ }^{5}$ La prueba Wald Z es poco confiable para muestras pequeñas, (Pardo et al., 2007; Hayes, 2006). Por tal razón, se incluye el indicador de contraste -2LL de verisimilitud restringida. La diferencia de este indicador entre dos modelos se distribuye como una Chi-cuadrado, con grados de libertad iguales a la diferencia de parámetros estimados. En este caso, $x^{2}(1)=9.68<0.05$. Por tanto, la estimación corrobora la estimación de la Wald Z. 
laboral explican un porcentaje equivalente de las discrepancias intraestatales de los IDH de los municipios. Por otro lado, el decremento en la dispersión del otro componente aleatorio refleja que la PML es responsable de 9,6\% de las diferencias interestatales de los IDH que, en promedio, registran las entidades federativas. En otros términos, la productividad laboral incide más en las discrepancias entre los estados que entre los municipios que éstos albergan. Si una entidad federativa logra desplegar actividades económicas de alta productividad en uno o en pocos de sus municipios provocará una mayor diferenciación entre el IDH medio estatal y el nacional que entre esos municipios y el resto de la entidad. En este sentido, se ahonda más la desigualdad entre estados que entre las localidades municipales del estado en cuestión.

\subsection{La inversión y el progreso técnico como variable de control: modelos 3 y 4}

La inversión es introducida como variable de control en el siguiente modelo, en virtud de que al dotar de más capital al trabajador, altera favorablemente la productividad media del trabajo y, por esta vía, el IDH. Lo mismo sucede con la productividad total de los factores (PTF). Para aproximar la inversión (I) se escogió una proxy que relaciona los activos fijos (AF) con el personal ocupado (L), mientras que para la PTF se utilizó una versión ligeramente modificada del Residuo de Solow: ${ }^{6}$

$$
\begin{gathered}
I_{i j}=\frac{A F_{i j}}{L_{i j}} \\
P F T_{i j}=V A_{i j}-[\alpha L+(1-\alpha) K] .
\end{gathered}
$$

Donde VA, representa el Valor Agregado Censal; y $\alpha$, la participación de los salarios en ese valor.

La introducción de ambas variables se realiza en forma secuencial, con el propósito de examinar de forma aislada sus efectos.

\subsubsection{La inversión}

Se trata de una variable del nivel 1, en virtud de que las inversiones tienen una dimensión local, y es en las comunidades en las cuales se asientan,

\footnotetext{
${ }^{6}$ En términos absolutos, el Residuo de Solow se estima como $P F T_{i j}=\Delta V A_{i j}-[\alpha \Delta L+(1-\alpha)$ $\Delta K]$. Sin embargo, se optó por sustituir las variaciones $(\Delta)$ por las valores finales, en virtud de que la información censal obligó a un análisis transversal, y no en otro, basado en series de tiempo.
} 
donde se espera que tenga el impacto deseado en términos de empleo, salarios e inscripción a la seguridad social.

El modelo se especifica de la siguiente manera:

$$
\begin{gathered}
I D H_{i j}=\beta_{0 j}+\beta_{1 j} P M L_{i j}+\beta_{2 j} I_{i j}+e_{i j} \text { (3.1) Nivel 1 } \\
\beta_{0 j}=\gamma_{00}+u_{0 j} ; \beta_{1 j}=\gamma_{10} ; \beta_{2 j}=\gamma_{10} \text { (3.2) Nivel 2 }
\end{gathered}
$$

La inversión opera como una variable de control, con el propósito de "aislar" el efecto de la PML sobre el IDH y neutralizar otras influencias. En realidad, ayuda a contestar el cuestionamiento de cómo influiría la PML en el IDH, si todos los municipios registraran la misma dotación de capital y, al mismo tiempo, la respuestas de la variable dependiente a esta última fueran idénticas para todos ellos $\left(\beta_{2 j}=\gamma_{10}\right)$

El modelo mixto es el siguiente:

$$
I D H_{i j}=\gamma_{00}+\gamma_{10} P M L_{i j}+\gamma_{20} I_{i j}+\left(u_{o j}+e_{i j}\right)
$$

La estimación correspondiente se encuentra en los cuadros 1 y 2 , en el rubro "modelo 3". En ellos se puede apreciar que PML e I son significativas. La media condicionada experimenta una alza ligera, a consecuencia de que se ha nulificado la injerencia de este último factor. Vale decir que si las dotaciones de capital por trabajador fueran similares en todos los municipios, sus IDH discreparían en 0,022 puntos por cada millón de pesos de diferencia en sus eficiencias laborales; es decir, en 2,7\% del promedio muestral incondicionado. Este resultado contrasta con el obtenido en el modelo vacío, en el que el impacto es de 0,027 puntos y de $3,3 \%$. En este sentido, la inversión parece ser responsable de 0,005 puntos, que equivale a 0,6 puntos porcentuales.

Los efectos aleatorios, en conjunto, no sufren alteración alguna: la varianza total permanece en el mismo nivel que en el modelo anterior. Pero se produce una mutación en su estructura: el aumento de la dispersión entre los estados es compensado exactamente por una variación inversa entre los municipios de una misma entidad federativa. La inversión, entonces, opera más como un dispositivo diferenciador intraestatal que interestatal. Es decir, los estados tienden a ser más homogéneos en sus promedios municipales de dotación por trabajador que los municipios entre sí. 


\subsubsection{El progreso técnico}

$\mathrm{Al}$ introducir el progreso técnico, aproximado por la PTF, los modelos de primer y segundo nivel, por un lado, y el mixto por el otro, se expresan de la siguiente manera:

$$
\begin{gathered}
I D H_{i j}=\beta_{0 j}+\beta_{1 j} P M L_{i j}+\beta_{2 j} I_{i j}+\beta_{3 j} P T F_{i j}+e_{i j}(4.1) \text { Nivel 1 } \\
\beta_{0 j}=\gamma_{00}+u_{0 j} ; \beta_{1 j}=\gamma_{10} ; \beta_{2 j}=\gamma_{10} \beta_{3 j}=\gamma_{30}(4.2) \text { Nivel } 2 \\
I D H_{i j}=\gamma_{00}+\gamma_{10} P M L_{i j}+\gamma_{20} I_{i j}+\gamma_{30} P T F_{i j}+\left(u_{o j}+e_{i j}\right)
\end{gathered}
$$

La estimación de los efectos fijos y de los aleatorios se encuentran en los cuadros 1 y 2 , en el rubro "modelo 4". La primera modificación es que la inversión ha dejado de ser significativa, mientras que la PTF sí lo es, aunque con signo negativo. El mensaje parece inequívoco: la primera estimula el desarrollo municipal siempre y cuando se introduzca sin progreso técnico ahorrador de mano de obra; es decir, se adapte a las calificaciones del trabajo local. Sin embargo, parece que el patrón de especialización asociado al modelo secundario-exportador tiende a demandar mano de obra calificada, que difícilmente es la que abunda en las localidades. Antes bien, tiende a desplazarla ya sea por otra más habilitada y mejor pagada, con un resultado neto negativo para el empleo y, probablemente, la masa salarial.

Algunos autores describen este mecanismo como el privilegiado, debido a que atiende los retos de la competencia global (Pizarro, 2001). En todo caso, éste parece ser válido para los primeros años de la apertura comercial, pero no para el periodo reciente. Si la mayor intensidad de capital, como hemos visto, aumenta el desarrollo municipal, un mayor progreso técnico en esta dirección no tendría por qué reducirlo; a menos que el patrón de especialización continúe con aquel tipo de demanda laboral, pero ésta sea crecientemente satisfecha con mano de obra calificada, pero más abundante y más barata, que desplaza a la mejor pagada -mayor a cinco salarios mínimos- con una peor remunerada. A esta conclusión se llega cuando se observa la nueva forma de precarización laboral, desde de la crisis de 2009 (Millán, 2013).

Los dos efectos aleatorios, por su parte, registran una varianza más reducida. Pero la PTF explica 2,63\% de las diferencias interestatales de los IDH y sólo $0,54 \%$ de las intraestatales. En conjunto, es responsable de $1 \%$ de ambas discrepancias. 


\subsection{La respuesta del desarrollo municipal a la productividad laboral: ¿diferencias?}

Los modelos multinivel suelen completar sus análisis con la inspección de las pendientes de las regresiones; es decir, con la posibilidad de que la respuesta de la variable dependiente (el IDH municipal) a sus determinantes (la productividad laboral y la inversión) sea distinta de un estado a otro. Como se puede advertir, los ejercicios anteriores suponían que esa respuesta, al ser fijada, era común en todas las entidades federativas. Sin embargo, no hay razón teórica para que esto sea así. Por tanto, es necesario examinar si existen diferencias en esas pendientes estatales. En caso de que así sea, se deberá introducir nuevas variables que ayuden a explicar las discrepancias. En caso contrario, el análisis termina ahí, en lo que a este tema se refiere.

La forma de hacerlo es similar al procedimiento del modelo vacío; la única diferencia es que ahora es la pendiente, y no sólo el intercepto, el que es sometido a efectos y aleatorios:

$$
\begin{gathered}
I D H_{i j}=\beta_{0 j}+\beta_{k j} V S_{i j}+e_{i j}(4.1 .1 .) \text { Nivel 1 } \\
\beta_{0 j}=\gamma_{00}+u_{0 j} ; \beta_{k j}=\gamma_{k 0}+u_{k j}(4.2 .1 .) \text { Nivel } 2 \\
I D H_{i j}=\gamma_{00}+\gamma_{k 0} V S_{i j}+\left(u_{o j}+u_{k j}+e_{i j}\right)(4.2 .3) \text { Mixto }
\end{gathered}
$$

Donde $V S_{i j}$ es la variable seleccionada entre PML, I y PTF; y k, el parámetro que le acompaña. Sin embargo, la estimación arrojó que ninguna de las varianzas de las pendientes consideradas fue significativa. ${ }^{7}$ Lo cual implica que las respuestas de los IDH a sus determinantes es similar entre los estados y, por tanto, no son fuente de diferenciación entre ellos.

\section{Instituciones y desarrollo municipal: la evidencia empírica}

Los resultados anteriores permiten concluir que las causas de discrepancias en los IDH ocurren entre los promedios estatales, y entre éstos y los registros municipales. Además, que son ocasionadas fundamentalmente por diferencias en las productividades laborales y totales, así como por la inversión. Sin embargo, se antoja que la porción explicada es todavía pequeña. En este sentido, es preciso introducir variables adicionales que permitan una mayor explicación. En términos estadísticos, ésta es la función que cumplirán las variables institucionales; en términos teóricos: validar o rechazar las hipótesis neoinstitucionalistas expuestas con anterioridad.

\footnotetext{
${ }^{7} \mathrm{La}$ varianza fue redundante para $u_{1 j}$. Para $u_{2 j}$, Wald $\mathrm{Z}=0.974 \mathrm{y}$ sig. $=0.330$; para $u_{3,}$, Wald $\mathrm{Z}=0$ y sig.=1. Además, la pruebas chi cuadrado de las diferencias de los $-2 \mathrm{LL}$ tampoco resultaron significativas.
} 


\subsection{Las variables institucionales}

Si algo no pudo cambiar la transición a la democracia fue la hegemonía de los gobiernos estatales sobre los municipales. Durante el periodo autoritario, ésta formaba parte del sistema centralista de dominación, que ubicaba al Ejecutivo federal en la cúspide de la pirámide del poder, y a la mecánica corporativista como la principal fuente de legitimidad (Millán, 1998). La democracia socavó ambos pilares del sistema político, pero robusteció el poder de los gobernadores, desprendidos ahora de los dispositivos disciplinarios implícitos en el viejo esquema. Esto les permitió reforzar su control sobre los municipios, ya fuera de forma directa o mediante la imposición de marcos institucionales, acicateados con la discrecionalidad suficiente para distanciarse de sus referentes federales. De esta manera, a la diversidad institucional de naturaleza informal, que provenía de circunstancias idiosincráticas, se sumó otra, originada en la mayor autonomía de los poderes estatales.

Por tal razón, se ha decidido insertar las variables en el segundo nivel: el estatal. Con este propósito se han construido tres indicadores, nutridos con la información que el IMCO utilizó para estimar los índices de competitividad de las entidades federativas en 2010. (IMCO, 2014). El primero de ellos es el Índice de Democratización (ID), cuya construcción es guiada por el concepto de "instituciones inclusivas" de Acemoglu y Robinson (2012), para denotar la inclusión de los ciudadanos en la dinámica económica, social y política, mediante esquemas democráticos. Los subíndices que lo integran son los siguientes indicadores del IMCO: Mercado Laboral; Sociedad Incluyente, Preparada y Sana; y Sistema Político Estable y Funcional. El segundo es Índice de Justicia y Gobierno (IJG), que aspira a dar cuenta del grado en que los gobiernos estatales hacen cumplir la ley y se apegan a ella. Está compuesto por tres indicadores de aquella organización: Sistema de Derecho Confiable y Objetivo; Gobierno Eficiente y Eficaz; y Precursores, que reporta obra y servicios públicos. El tercero es el Índice de Innovación (INO), que se incluye textualmente desde la información del IMCO. ${ }^{8}$

Éste construyó cada uno de estos indicadores mediante una escala normalizada de cero a cien, en la que la entidad mejor posicionada recibe la calificación más alta, y la peor, la más baja. Por tanto, las variables institucionales que se proponen en este ejercicio varían en el mismo rango, porque representan los promedios simples de sus ingredientes. (Los dos últimos indicadores atienden, por supuesto, al énfasis de North sobre

\footnotetext{
${ }^{8}$ Por razones de espacio, se invita al lector interesado a consultar el contenido de estos "subíndices" en la base de datos del IMCO (2014).
} 
el cumplimiento de los contratos, el imperio de la ley y la innovación, cada uno de ellos vinculados a los derechos de propiedad).

\subsection{Los determinantes institucionales (Modelos 5, 6 y 7 )}

En esta sección se despliegan tres modelos alternativos, pero complementarios, que incorporan secuencialmente las variables institucionales.

\subsubsection{La influencia de la democratización: modelo 5}

En el primer modelo se deja operar al Índice de Democratización como determinante de los IDH municipales; o, más precisamente, como presunto responsable de las discrepancias que éstos guardan con relación a sus promedios estatales, y de las que prevalecen entre estos últimos y las demás entidades federativas. De esta forma, el modelo se especifica de la siguiente manera:

$$
\begin{gathered}
I D H_{i j}=\beta_{0 j}+\beta_{i j} P M L_{i j}+\beta_{2 j} I_{i j}+\beta_{3 j} P T F_{i j}+e_{i j} \text { (5.1) Nivel 1 } \\
\beta_{0 j}=\gamma_{00}+\gamma_{01} I D_{j}+u_{0 j} ; \beta_{1 j}=\gamma_{10} ; \beta_{2 j}=\gamma_{20} ; \beta_{3 j}=\gamma_{30} \text { (5.2.) Nivel } 2
\end{gathered}
$$

El ID, como todas las variables institucionales, ha sido centrado por su media; por tanto, el intercepto $\gamma_{00}$ continúa representando "la gran media"; $\beta_{0 j}$, la estatal; y el término aleatorio $u_{0 j}$, la diferencia entre ambas, pero exenta de la influencia del ID; es decir, de $\gamma_{01} I D_{j}$. Otra forma de expresarlo: las discrepancias entre los promedios estatales y nacionales manifiestan las desviaciones en los IDH, debidas tanto a los determinantes del primer nivel como al grado de democratización: $\beta_{0 j}=\gamma_{00}+\gamma_{01} I D_{j}+u_{0 j}$;

El modelo mixto es el siguiente:

$$
I D H_{i j}=\gamma_{00}+\gamma_{01} I D_{j}+\gamma_{10} P M L_{i j}+\gamma_{20} I_{i j}+\gamma_{30} P T F_{i j}+\left(u_{o j}+e_{i j}\right)
$$

Los cálculos se reportan en los cuadros 1 y 2 en el rubro de "modelo 5 ". En ellos se aprecia que el coeficiente de ID es significativo y con signo positivo. Esto significa que un mayor grado de democratización de las entidades federativas se traduce en más desarrollo municipal, como afirma la tesis de Acemoglou y Robinson (2012). Para comprender mejor este resultado, es conveniente analizar lo que sucede con los efectos aleatorios. Ahí se puede observar que la varianza de la intersección, $\mathrm{UN}(1,1)$, ha dejado de ser significativa, mientras que la del ID, UN $(2,2)$, es redundante. Es decir, todas las diferencias entre los promedios estatales de IDH pueden ser atribuidas a las discrepancias que, entre las entidades federativas, prevalecen en el grado de democratización, una vez que se descuenta, 
por supuesto, la influencia de las productividades laboral y total. ${ }^{9}$ El término refleja íntegramente esas diferencias porque se constituye en la distancia entre las medias estatales y la media muestral de los IDH.

El efecto fijo implica que si dos entidades federativas difieren en 10 unidades del Índice de Democratización, también lo harán en 0,03 puntos los IDH municipales, que equivale a 3,5\% de la media muestral incondicionada.

Otra forma de apreciar el efecto del ID sobre el desarrollo municipal consiste en contrastar su coeficiente con el de la PML y observar la reducción en la varianza total. El primer aspecto revela que el ID tiene un impacto 158 veces mayor que el de la productividad laboral. El segundo nos dice que esta variable redujo 4,7\% la varianza total; la PTF, 1,27\%; I, 0\%; y el ID, 33,8\%. Estas cifras corresponden al grado de explicación de las diferencias entre los IDH municipales de cada una de estas variables.

\subsubsection{La influencia del Gobierno y la Justicia: modelo 6}

Como se ha expuesto antes, la influencia de la justicia para hacer cumplir los contratos y la alineación de los intereses del personal del gobierno con los incentivos que promueven el desarrollo, son instituciones reconocidas por el neoinstitucionalismo, aunque enfatizadas por North (1984 y 1993). En este sentido, se incorpora la variable IGJ en segundo nivel, que es el que corresponde a los estados. El modelo se especifica de la siguiente manera:

$$
\begin{aligned}
& I D H_{i j}=\beta_{0 j}+\beta_{1 j} P M L_{i j}+\beta_{2 j} I_{i j}+\gamma_{20} I_{i j}+\beta_{3 j} P T F_{i j}+e_{i j} \text { (6.1) Nivel 1 } \\
& \beta_{0 j}=\gamma_{00}+\gamma_{02} I J G_{j}+u_{0 j} ; \beta_{1 j}=\gamma_{10} ; \beta_{2 j}=\gamma_{20} ; \beta_{3 j}=\gamma_{30}(6.2) \text { Nivel } 2
\end{aligned}
$$

El modelo mixto, por tanto, es:

$$
I D H_{i j}=\gamma_{00}+\gamma_{02} I J G_{j}+\gamma_{10} P M L_{i j}+\gamma_{20} I_{i j}+\gamma_{30} P T F_{i j}+\left(u_{o j}+e_{i j}\right)
$$

Los resultados se reportan en el "modelo 6" de los cuadros 1 y 2 . En el primero de ellos se advierte que IJG no tiene efectos fijos, en virtud de no es significativo. Pero los efectos aleatorios sí lo son, especialmente los que involucran la intersección y los residuos. $\mathrm{Al}$ ser $\gamma_{02} I J G_{j}=0$, éstas se expresan como $\beta_{0 j}-\gamma_{00}=u_{o j}$; pero si el efecto aleatorio de la varianza de IJG, $\mathrm{UN}(2,2)$ es redundante y la covarianza $\mathrm{UN}(2,1)$ no es significativa, entonces toda la varianza de $u_{o j}$ recae en las discrepancias intra e interestatales, ligadas a UN $(1,1)$. En conjunto, ellos reducen la varianza total en

${ }^{9} \mathrm{La}$ PTF ha dejado de ser significativa. Por otro lado, la diferencia entre los -2LL del modelo 4 y 5 , arroja que $\chi^{2}(1)=0.036<0.05$. 
$8,11 \%$, pero la mayor parte de este decremento reposa en diferencias interestatales de las medias en los IDH. Por tanto, se concluye el IJG ejerce una influencia entre los IDH promedio interestatales a través de efectos aleatorios. Otra forma de decirlo: la justicia y el gobierno impactan el desarrollo municipal mediante particularidades estatales, sin que existan efectos similares entre un estado y otro, como lo sugeriría la existencia de efectos fijos. En segundo término, esta variable es la tercera en importancia (después del ID e INO, como se verá) al explicar las discrepancias en el desarrollo de los municipios; superior, incluso, que la productividad laboral.

\subsubsection{La influencia de la innovación}

Por último, se introduce la variable institucional que da cuenta de la invocación. Los modelos por niveles y el mixto son los siguientes:

$$
\begin{aligned}
& I D H_{i j}=\beta_{0 j}+\beta_{1 j} P M L_{i j}+\beta_{2 j} I_{i j}+\beta_{3 j} P T F_{i j}+e_{i j} \text { (7.1) Nivel 1 } \\
& \beta_{0 j}=\gamma_{00}+\gamma_{02} I N O_{j}+u_{o j} \beta_{1 j}=\gamma_{10} ; \beta_{2 j}=\gamma_{20} ; \beta_{3 j}=\gamma_{30}(7.2) \text { Nivel 2 } \\
& I D H_{i j}=\gamma_{00}+\gamma_{02} I J G_{j}+\gamma_{10} P M L_{i j}+\gamma_{20} I_{i j}+P T F_{i j}+\left(u_{o j}+e_{i j}\right) \text { Mixto (7.3) }
\end{aligned}
$$

Los resultados los consigna el rubro "modelo 7" de los cuadros 1 y 2. Un aspecto es, por demás, interesante: los efectos fijos y aleatorios, con excepción de los residuos, no son significativos. Esto implica que $\beta_{0 j}=\gamma_{00}$; es decir, que esta variable institucional no determina diferencias interestatales en el desarrollo municipal. Sólo ejerce su influencia en las discrepancias intraestatales. De hecho, contribuye a reducir la varianza total en $32,5 \%$, una cifra ligeramente inferior a la correspondiente al ID. La distinción entre ambas, es que esta última variable lo hace en el ámbito interestatal, mientras que INO ejerce su influencia en la esfera intraestatal.

\section{Conclusiones}

Por tanto, se puede concluir que las variables institucionales son las que registran mayor poder de explicación de las diferencias en el desarrollo municipal, seguidas de las productividades laboral y total de los factores. Entre ellas, el grado de democratización y la innovación son, en ese orden, las más importantes. Pero mientras la primera ejerce principalmente su influencia en las discrepancias interestatales, la segunda, la activa en el plano intraestatal. El impacto del ID se despliega mediante efectos fijos -y por tanto comunes a todos los municipios-y aleatorios (o específicos), mientras la innovación sólo a través de estos últimos. El sistema de justicia y de gobierno también fue relevante al constituirse en un determinante de las discrepancias, pero sustancialmente menor que las otras dos variables 
institucionales, lo que parece confirmar la superioridad de la tesis de Acemoglou y Robinson (2012) sobre la de North (1984 y 1993), aunque ésta también se ve avalada por los datos.

Por último, la búsqueda de las causas profundas del desarrollo encuentra una respuesta satisfactoria en el neoinstitucionalismo, con independencia de cuál sea el énfasis que pone sobre una u otra causal institucional, porque son los factores de esta índole los que exhiben un mayor potencial de explicación que los determinantes más inmediatos y más invocados por los analistas: los factores económicos.

\section{Cuadro 1 \\ Efectos fijos}

\begin{tabular}{|c|c|c|c|c|}
\hline Modelo & Estimación & Error típico & pruebat & Sig. \\
\hline \multicolumn{5}{|l|}{ 1) Vacío } \\
\hline Intersección & 0,815978 & 0,007033 & 116,0 & 0,000 \\
\hline \multicolumn{5}{|l|}{ 2) Modelo 2} \\
\hline Intersección & 0,814188 & 0,006751 & 120,6 & 0,000 \\
\hline PML & 0,000027 & 0,000008 & 3,5 & 0,001 \\
\hline \multicolumn{5}{|l|}{ 3) Modelo 3} \\
\hline Intersección & 0,814231 & 0,006784 & 120,0 & 0,000 \\
\hline PML & 0,000022 & 0,000008 & 2,8 & 0,006 \\
\hline I & 0,000007 & 0,000004 & 1,99 & 0,047 \\
\hline \multicolumn{5}{|l|}{ 4) Modelo 4} \\
\hline Intersección & 0,812299 & 0,006767 & 120 & 0,000 \\
\hline PML & 0,000021 & 0,000008 & 2,7 & 0,008 \\
\hline I & 0,000004 & 0,000004 & 1,09 & 0,275 \\
\hline PTF & $-3,21 \mathrm{E}-10$ & $1,55 \mathrm{E}-10$ & $-2,07$ & 0,040 \\
\hline \multicolumn{5}{|l|}{ 5) Modelo 5} \\
\hline Intersección & 0,79422 & 0,007087 & 112 & 0,000 \\
\hline PML & 0,000019 & 0,000008 & 2,4 & 0,015 \\
\hline I & 0,000005 & 0,000004 & 1,2 & 0,215 \\
\hline PTF & $-2,23 \mathrm{E}-10$ & $1,34 \mathrm{E}-10$ & $-1,7$ & 0,097 \\
\hline ID & 0,002987 & 0,000588 & 5,1 & 0,000 \\
\hline \multicolumn{5}{|l|}{ 6) Modelo 6} \\
\hline Intersección & 0,80764 & 0,007187 & 112,4 & 0,000 \\
\hline PML & 0,000021 & 0,00001 & 2,6 & 0,009 \\
\hline I & 0,000004 & 0,000004 & 1,1 & 0,277 \\
\hline PTF & $-3,16 \mathrm{E}-10$ & $1,53 \mathrm{E}-10$ & $-2,1$ & 0,040 \\
\hline IJG & 0,002093 & 0,001092 & 1,9 & 0,065 \\
\hline \multicolumn{5}{|l|}{ 7) Modelo 7} \\
\hline Intersección & 0,810305 & 0,006438 & 125,9 & 0,000 \\
\hline PML & 0,000021 & 0,000008 & 2,6 & 0,009 \\
\hline I & 4,35E-06 & 3,77E-06 & 1,15 & 0,249 \\
\hline PTF & $-3,03 \mathrm{E}-10$ & $1,47 \mathrm{E}-10$ & $-2,06$ & 0,041 \\
\hline INO & 0,001130 & 0,000571 & 1,98 & 0,175 \\
\hline
\end{tabular}

Fuente: elaboración propia con datos de PNUD, 2010; Inegi, 2009; IMCO, 2014. 


\section{Cuadro 2}

Estimación de parámetros de covarianza

\begin{tabular}{|c|c|c|c|c|}
\hline Modelo & Estimación & Error tipico & Wald Z & Sig. \\
\hline \multicolumn{5}{|l|}{ 1) Vacío } \\
\hline Residuos & 0,002079 & 0,000138 & 15,06 & 0,000 \\
\hline Varianza de $\mathrm{u}_{0 \mathrm{j}}$ (estado) & \multicolumn{4}{|c|}{$-2 \mathrm{LL}=-1543,67$} \\
\hline \multicolumn{5}{|l|}{ 2) Modelo 2} \\
\hline Residuos & 0,00204 & 0,000136 & 15,04 & 0,000 \\
\hline Varianza de $\mathrm{u}_{0 \mathrm{j}}$ (estado) & \multicolumn{4}{|c|}{$-2 \mathrm{LL}=-1533,99$} \\
\hline \multicolumn{5}{|l|}{ 3) Modelo 3} \\
\hline Residuos & 0,002025 & 0,000135 & 15,02 & 0,000 \\
\hline Varianza de $\mathrm{u}_{0 \mathrm{j}}$ (estado) & \multicolumn{4}{|c|}{$-2 \mathrm{LL}=-1514,67$} \\
\hline \multicolumn{5}{|l|}{ 4) Modelo 4} \\
\hline Residuos & 0,002014 & 0,000134 & 15,02 & 0,000 \\
\hline Varianza de $\mathrm{u}_{0 \mathrm{j}}$ (estado) & \multicolumn{4}{|c|}{$-2 \mathrm{LL}=-1475,60$} \\
\hline 5) Modelo 5 & & & & \\
\hline Residuo & 0,002015 & 0,000134 & 15,1 & 0,000 \\
\hline $\mathrm{UN}(1,1)$ & 0,000659 & 0,000358 & 1,8 & 0,066 \\
\hline $\mathrm{UN}(2,1)$ & $-0,000038$ & 0,000014 & $-2,7$ & 0,008 \\
\hline $\mathrm{UN}(2,2)$ & \multicolumn{4}{|c|}{$-2 \mathrm{LL}=-1480,087$} \\
\hline \multicolumn{5}{|c|}{$\begin{array}{l}\text { Intercept }+ \text { ID_C }[\text { subject }= \\
\text { Estado }]\end{array}$} \\
\hline \multicolumn{5}{|c|}{$\begin{array}{l}\text { b This covariance parameter is redundant. The test statistic and confidence interval } \\
\text { cannot be computed. } \\
\text { 6) Modelo } 6\end{array}$} \\
\hline Residuo & 0,002013 & 0,000134 & 15,0 & 0,000 \\
\hline $\mathrm{UN}(1,1)$ & 0,000985 & 0,000401 & 2,5 & 0,014 \\
\hline $\mathrm{UN}(2,1)$ & $-0,000023$ & 0,000046 & $-0,512$ & 0,609 \\
\hline $\mathrm{UN}(2,2)$ & $\begin{array}{r}5,605542 \mathrm{E}- \\
7 \mathrm{~b}\end{array}$ & 0,000000 & . & . \\
\hline $\begin{array}{l}\text { Intercept + IJG_C [subject } \\
=\text { Estado }]\end{array}$ & \multicolumn{4}{|c|}{$\begin{array}{l}\text { b This covariance parameter is redundant. The test statistic and confidence interval } \\
\text { cannot be computed. }\end{array}$} \\
\hline \multicolumn{5}{|c|}{$-2 \mathrm{LL}=-1468,60$} \\
\hline \multicolumn{5}{|l|}{ 6) Modelo 6} \\
\hline Residuo & 0,002019 & 0,000135 & 15,0 & 0,000 \\
\hline $\mathrm{UN}(1,1)$ & 0,000772 & 0,000464 & 1,7 & 0,096 \\
\hline $\mathrm{UN}(2,1)$ & $-0,000021$ & 0,000019 & $-1,1$ & 0,265 \\
\hline $\mathrm{UN}(2,2)$ & 0,000002 & 0,000004 & 0,43 & 0,668 \\
\hline \multicolumn{5}{|c|}{ Intercept + INO_C [subject = Estado $]$} \\
\hline
\end{tabular}

Fuente: elaboración propia con datos de PNUD, 2010; Inegi, 2009; IMCO, 2014. 


\section{Fuentes consultadas}

Acemoglu, Daron y James Robinson (2012), Why nation fail. The origins of prosperity, power, and poverty, Crown Publisher, New York.

Acemoglu Daron, Simon Johnson y James Robinson (2001), "The colonial origins of comparative development: an empirical investigation", The American Economic Review, 91 (5), American Economic Association, New York, pp. 1369-1401.

Alchian, Armen A. (1950), "Uncertainty, evolution and economic theory", Journal of Political Economy, núm. 58, Chicago University, Chicago, pp. 211-221.

Gallup, John Luke, Jeffrey Sachs y Andrew Mellinger (1998), "Geography and economic develoment", National Bureau of Economic Research, working paper, WP 6849, Cambridge, pp. 1-81.

Hayes, Andrew F. (2006), "A primer on multilevel modeling", Human Communication Research", núm. 32, International Communication Association, Washington, pp. 385-410.

Inegi (2009), Censos económicos, consulta interactiva de datos, <http:// www.inegi.org.mx/est/contenidos/espanol/proyectos/censos/ ce/2009/default.asp?c=1422>, diciembre 2014.

IMCO (2014), "Indice de competitividad estatal 2014. Las reformas y los estados. Base de datos de 2010", http://imco.org.mx/indices/\#!/ competitividad_estatal_2014/análisis, 29 de diciembre 2014.

Koutsoyianis, A. (1985), Microeconomía moderna, Amorrortu, Buenos Aires.

Lucas, Robert E. (1988), "On the mechanics of economic development", Journal of Monetary Economics, 22 (1), Carnegie Mellon University, Pittsburgh, pp. 3-42.

Millán, Henio (2013), En la cuerda floja. Vulnerabildad hacia la pobreza y fragilidad laboral en México, El Colegio Mexiquense, A. C., Toluca.

Millán, Henio (2012), Politica y desarrollo. Las instituciones en sociedades heterogéneas, El Colegio Mexiquense, A. C., Toluca. 
Millán, Henio (1998), Neoliberalismo y transición en México, El Colegio Mexiquense, A. C., Toluca.

North, Douglas (1993), Instituciones, cambio institucional y desempeño económico, Fondo de Cultura Económica, México.

North, Douglas (1984), Estructura y cambio en la historia económica, Alianza, Madrid.

Pardo, Antonio, Miguel Angel Ruiz y Rafael San Martín (2007), “Como ajustar e interpretar modelos multinivel con SPSS", Psicothema, 19 (2), Universidad de Oviedo, Oviedo, pp. 308-321.

Pizarro, Roberto (2001), La vulnerabilidad social y sus desafios: una mirada desde América Latina, serie Estudios estadísticos y prospectivos, núm. 6 (LC/L.1490-P), Comisión Económica para América Latina y el Caribe (CEPAL), Santiago de Chile.

PNUD (2010), "Índice de desarrollo humano municipal”, <http://goo. $\mathrm{gl} / \mathrm{jpcvYq}>, 15$ de septiembre de 2015.

Ricardo, David (1817 y 1998), Principios de economía politica y tributación, Fondo de Cultura Económica, México.

Rodrik, Dani, Arvind Subramanian y Francesco Trebbi (2002), "Institutions rule: the primacy of institutions over integration and geography in economic development", International Monetary Fund, working paper, 02/189, International Monetary Fund, Washington, pp. $1-47$.

Romer, Paul (1994), "The origins of endogenous growth", Journal of Economic Perspectives, 8 (1), American Economic Association, New York, pp. 3-22.

Solow, Robert (1956), "A contribution to the theory of economic growth", Quarterly Journal of Economics, MIT Press, Boston, pp. 65-94. 
Henio Millán-Valenzuela. Mexicano. Doctor en ciencias sociales por la Universidad Iberoamericana; maestro en economía por el CIDE y licenciado en historia por la UAM-Iztapalapa. Recibió el Premio Estatal de Ciencia y Tecnología, 2014, otorgado por el Consejo Mexiquense de Ciencia y Tecnología. Su artículo "Las causas de la crisis financiera" obtuvo el primer lugar como el artículo más leído en la historia de la revista Economía, Sociedad y Territorio, en 2011. Sus líneas actuales de investigación son pobreza y desarrollo, así como democracia e instituciones. Ha publicado nueve libros y más de 40 artículos en revistas especializadas. Entre sus libros más recientes se encuentran: En la cuerda floja. Vulnerabilidad hacia la pobreza y fragilidad laboral, El Colegio Mexiquense, A. C., Toluca (2013); Politica y desarrollo. Las instituciones en sociedades heterogéneas, El Colegio Mexiquense, A. C., Toluca, (2012); Pobreza y Microfinanzas. Una evaluación de impacto, El Colegio Mexiquense, A. C., Toluca (2009); en coautoría: Participación y abstencionismo electoral en México, Instituto Federal Electoral-Universidad Autónoma de Querétaro, México (2011). Entre sus artículos destacan: "Los efectos crecimiento y redistribución: una propuesta metodológica. Ciclo económico y pobreza en México", El Trimestre Económico, LXXXI (323), Fondo de Cultura Económica, México, pp. 655-685 (2013); "El modelo democrático en México: entre la pre y la postmodernidad", Economía, Sociedad y Territorio, XIII (42), El Colegio Mexiquense, A. C., Toluca, pp. 459-494 (2013); "La democracia secuestrada. Actores sociales y representatividad en México”, Perfiles Latinoamericanos, núm. 40, Facultad Latinoamericana de Ciencias Sociales, México, pp. 165-204 (2012).

Martha Paola García-Pérez. Mexicana. Maestra en ciencias sociales con especialidad en desarrollo municipal en el Colegio Mexiquense, A. C., Licenciada en economía por la Universidad Autónoma del Estado de México. Fue becaria de investigación por la Universidad Autónoma del Estado de México en conjunto con la Universidad del País Vasco (UPV) (2010-2011); dicha investigación con clave 2873/2010. Actualmente es profesora en la Facultad de Economía de la Universidad Autónoma del Estado de México y en la Universidad campus Siglo XXI. 